This item was submitted to Loughborough's Research Repository by the author.

Items in Figshare are protected by copyright, with all rights reserved, unless otherwise indicated.

\title{
Antenna performance on quasi synthetic media
}

PLEASE CITE THE PUBLISHED VERSION

http://dx.doi.org/10.1109/IWAT.2012.6178672

PUBLISHER

(c) IEEE

VERSION

AM (Accepted Manuscript)

LICENCE

CC BY-NC-ND 4.0

REPOSITORY RECORD

Njoku, Chinwe C., W.G. Whittow, and J.C. Vardaxoglou. 2019. "Antenna Performance on Quasi Synthetic Media". figshare. https://hdl.handle.net/2134/9991. 
This item was submitted to Loughborough's Institutional Repository (https://dspace.lboro.ac.uk/) by the author and is made available under the following Creative Commons Licence conditions.

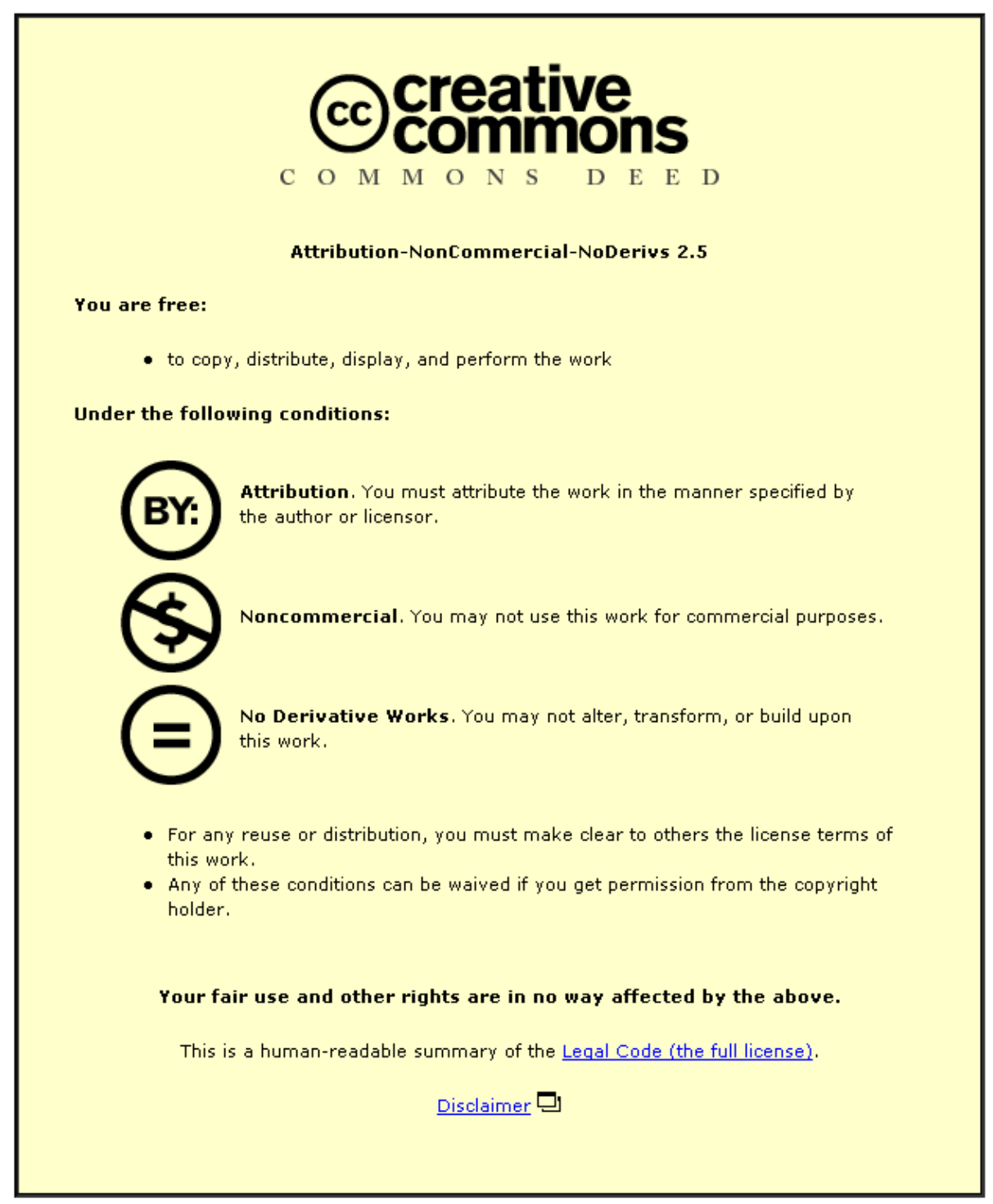

For the full text of this licence, please go to: http://creativecommons.org/licenses/by-nc-nd/2.5/ 


\title{
Antenna Performance on Quasi Synthetic Media
}

\author{
Invited paper \\ Chinwe C. Njoku $^{(1)}$, William G. Whittow ${ }^{(1)}$, and Yiannis C. Vardaxoglou ${ }^{(1)}$ \\ (1) School of Electronic, Electrical and Systems Engineering, Loughborough University, Loughborough, LE11 $3 T U$. \\ UK \\ Email: c.c.njoku@lboro.ac.uk; w.g.whittow@lboro.ac.uk; j.c.vardaxoglou@lboro.ac.uk
}

\begin{abstract}
While we seem to be experiencing a material evolution by applying unique properties of metamaterials, such as negative constitutive parameters and to some extent cloaking phenomena, not much attention has been paid in the practical suitability of synthetic materials towards antenna designs. The antenna designer is often faced with a judicious choice of:- complexity in the conducting/radiating shape, substrate and radome parameters, cost as well as ever increasing environmental effects both in the construction but also in the disposal of the antenna as part of a recycling process. This paper will outline some of the hypotheses and processes that underpin our terminology of quasi synthetic media and will proceed to illustrate how one can obtain a variety of dielectric (and magnetic) effective contrasts from 3-D structures containing either dielectric or conducting micro particles. Some representative patch designs are considered to indicate how one could replace cumbersome conventional design and manufacturing processes by using nanotechnology and additive manufacturing.
\end{abstract}

\section{INTRODUCTION}

In recent years, wires have been increasingly replaced by wireless alternatives. To achieve this, high performance antennas must be incorporated into a range of products including mobile phones, tablet computers and headsets. Despite this, the general public are not interested in the antennas and often do not even know their gadgets contain antennas. Therefore, the antennas must be hidden and also operate in more cluttered environments and on smaller platforms. While other electronics continue to decrease in size, it is not straightforward to create smaller antennas with the same level of electromagnetic performance. Fundamental physics dictate that the maximum efficiency, bandwidth and gain are related to the size of the sphere containing the antenna [1-3]. There has been a huge amount of work in the literature investigating different antenna geometries, including slots to reduce the size. The microstrip patch antenna has received a lot of attention because of its simplicity and low profile [4-6]. In the last 15 years, advancements in electromagnetic software allow thousands of different geometries to be investigated and further improvements to antenna design are becoming ever smaller. We are approaching a point when it will no longer be possible to make improvements by changing the geometry alone and we need to start considering optimizing the material properties and composition.

Patch antennas on printed circuit boards (PCB) are popular designs. However, these antennas require numerous processes including applying the photoresist and then etching away the unwanted copper. This creates several areas of inefficiency and increased cost: a) copper is wasted; b) environmentally damaging chemicals are required and c) many distinct machines are needed which takes up a lot of real estate and d) the seperate processes require a lot of manual intervention and approximately eight hours of processing time. Even though antennas are intrinsic to the wireless world, a typical cell phone antenna must be made for a fraction of a dollar. As material and labor prices continue to increase, costs must be decreased elsewhere. Furthermore, there will be increasing political, public and economic pressure to consider and implement environmentally beneficial fabrication systems. This suggest that replacing destructive manufacturing techniques with additive manufacturing techniques $[7,8]$ is beneficial.

Antenna designers are often restricted by the limited commercially available substrates and are forced to design their structures using specific pre-defined permittivities, losses and substrate heights. These options are unlikely to be the optimal choice from an electromagnetic perspective. High quality substrates with low losses and high permittivities can cost hundreds of dollars which means they are often not a practical option. Therefore, developing a technology that would allow the antenna designer to control the dielectric material and thickness would increase the freedom of their designs. By moving away from conventional PCBs, 3-D antennas can be created which fill more of the radian sphere than conventional 2-D patch antennas and hence improve the efficiency-bandwidth product. 
Electromagnetic advantages including increased bandwidth can be achieved by using textured dielectrics where the permittivity varies across the substrate. Specifically this is achieved by locating a low permittivity region where the electric fields are largest and vice-versa [9-12]. The authors have previously showed that a small size antenna with high bandwidth and efficiency can be designed using a substrate with equality of the relative permeability and permittivity [13]. At microwave frequencies, the losses of currently available magnetic materials are prohibitive but will be realizable in the future via synthetic manufacturing processes.

The authors believe that nanotechnology could be the solution. Nanoparticles have a very large surface area to volume ratio and hence they have amazing properties such as increased conductivity, increased strength and scratch resistance to name but a few. These novel properties have sparked billions of dollars of governmental and commercial investment and consequently the technology is advancing at a rapid rate, for example see http://www.youtube.com/watch?v=T8p8_zZNJEU.

The points above demonstrate that fabricational and electromagnetic advantages are possible by developing new manufacturing processes which allow the material properties to be synthetically controlled. An entire antenna system including the transmission line, substrate, via, patch and radome can be potentially made in one integrated process. This can be done with additive technology using only two materials: metallic cuboid (shown in black) and a host dielectric material (green) in Figure 1 (a). Each cuboid would be on the micro-scale and be composed of nanomaterials. The micro-scale cuboids can then couple to form structures on the millimetre/centimeter scale to function at microwave frequencies. Heterogeneous substrates can be produced by varying the local density of the metallic cuboids. Note, the permittivity can then be varied in all three dimensions.
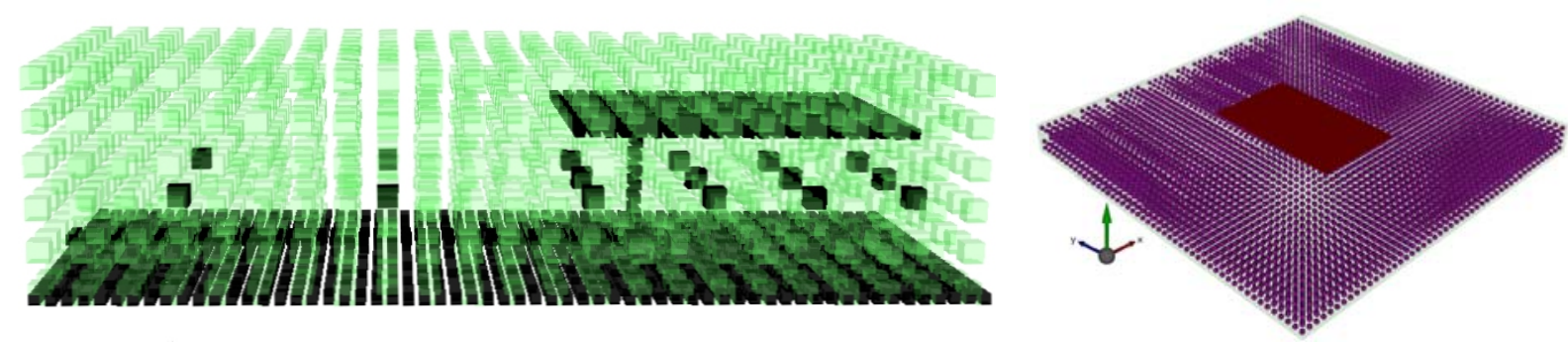

Figure 1. (a) A sketch (not to scale) showing how an antenna could be designed using quasi-synthetic media; (b) A simulated patch antenna with a substrate containing heterogeneous spherical inclusions

\section{HETEROGENEOUS SUBSTRATES WITH INCLUSIONS}

It has been theoretically known for many decades that inserting obstacles that are much smaller than a wavelength in a host medium can change the effective permittivity [14]. The inserted objects could be a distinct dielectric material or a metallic object. Lewin is the most often cited paper in this area but other authors have also analyzed the problem and the different analytical equations are compared in [15]. The analytical theory generally considers spherical particles embedded in a cubic lattice for simplicity however, cubic inclusions would also produce the same effect. Therefore, a higher effective permittivity could be achieved by increasing the volume density of the inclusions.

A general mathematical expression for the effective electromagnetic properties of particle-embedded mixtures [16] is given in equation (1) where $\mathrm{K}_{1}, \mathrm{~K}_{2}$ and $\mathrm{K}_{\mathrm{eff}}$ are the appropriate parameters $(\varepsilon, \mu, \sigma)$ of the host medium, the inclusions and the mixture respectively; $p$ is the total volume fraction of the inclusions, based on the local unit cell of the medium.

$$
K_{\text {eff }}=K_{1} \frac{\left[\left(K_{2}+2 K_{1}\right)\left(K_{1}-K_{2}\right)^{-1}\right]-2 p+C\left(K_{1}, K_{2}, p\right)}{\left[\left(K_{2}+2 K_{1}\right)\left(K_{1}-K_{2}\right)^{-1}\right]+p+C\left(K_{1}, K_{2}, p\right)}
$$

The behavior of these heterogeneous mixtures was examined in great detail in [17]. A simulation methodology to find the mixture's effective permittivity was implemented. The scattering parameters of a plane wave excitation incident of the heterogeneous substrate were processed using a Resonant Inverse Scattering Formalism [18]. This methodology was initially verified by analyzing homogeneous substrates [19]. The electromagnetic simulations showed good agreement with the canonical equations. The paper showed that the spherical inclusions could be replaced with cubic inclusions 
and produced similar permittivity values as long as the inclusion volume ratio was the same [17]. Metals have a very high equivalent permittivity and hence the use of metallic inclusions can lead to higher effective permittivities. Scaled waveguide measurements of metallic spheres embedded in a dielectric showed good agreement with the simulations and the analytical equations [17].

Theory suggests that there is an upper limit for the ratio of the effective permittivity to the host permittivity dependent on the volume fraction. For spheres in a simple cubic lattice, the maximum volume fraction is $\sim 0.5$ and hence the effective permittivity of the mixture can have a maximum value of 4.297 times the host value. However, the volume fraction limit is relaxed when cubic inclusions are used instead of spherical inclusions. Relative increases of more than twenty times were found for closely packed cubic inclusions, see Figure 2(a) [20]. Samples with cuboid inclusions were assembled and measurements of the permittivity with a resonator showed good agreement with the simulations. The simulations in $[17,20]$ showed that the effective loss tangent of the mixture was similar to the host medium. The measurements also indicated that the losses were not significantly increased by the use of the inclusions.
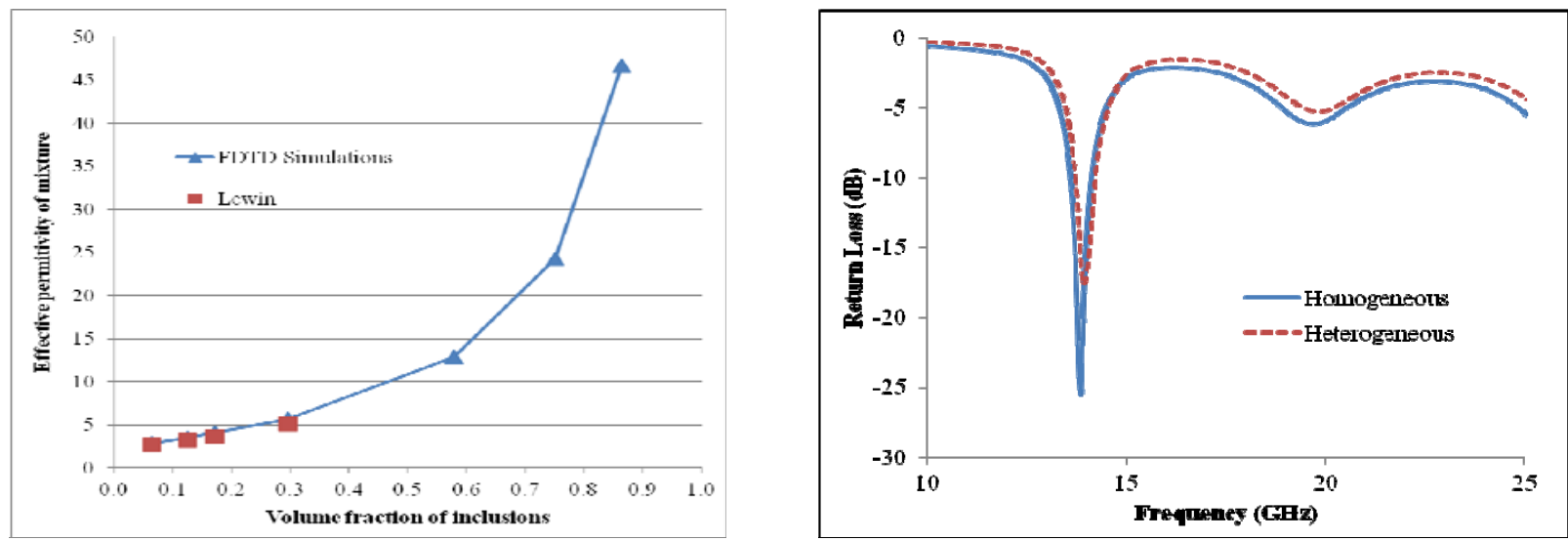

Figure 2. (a) Comparison of simulated and analytical effective permittivity as a function of volume fraction; (b) Return loss results for a simulated homogeneous patch and patch with small spherical inclusions

\section{ANTENNA DESIGN}

These materials with inclusions can be used to control the local permittivity of antenna substrates. EMPIRE Xcel Finitedifference time-domain (FDTD) was used to examine a patch antenna with 150micron cubes embedded in a host [17], see Figure 1 (b). A patch antenna with a heterogeneous substrate was simulated using a host material $\left(\varepsilon_{\mathrm{r}}=3.55\right.$, loss tangent $=0.0027)$ and $150 \mu \mathrm{m}$ diameter inclusion spheres $\left(\varepsilon_{\mathrm{r}}=11.9\right.$, loss tangent $\left.=0.01\right)$ with $250 \mu \mathrm{m}$ separation. This 50 x $30 \mathrm{~mm}$ patch antenna resonated at $13.8 \mathrm{GHz}$ which agreed closely with the homogeneous patch (where the permittivity of $\left(\varepsilon_{\mathrm{r}}=4.108\right.$ and loss tangent $\left.=0.008711\right)$ were calculated using the plane wave simulations of the substrate alone $)$, see Figure 2(b). The efficiency of the heterogeneous patch was $85 \%$ compared to $87 \%$ for the homogeneous case. Additionally, the coupling between two adjacent heterogeneous patch antennas showed good agreement with two patches on a homogeneous substrate which indicated that the surface waves were not adversely affected. This provides further confidence that these quasi-synthetic media can be used as antenna substrates in realistic conditions.

In [21] patch antennas above substrates with small scale inclusions where the effective permittivity varied locally were examined. The simulated efficiencies were similar to the homogenous patch antenna. Controlling the local permittivity, altered the currents and electric fields and hence the antenna performance. A dual-band design was instigated with the use of heterogeneous substrates.

\section{SUMMARY AND FUTURE WORK}

Developing quasi-synthetic media has the potential to be advantageous both fabricationally and electromagnetically. Simulations, measurements and analytical theory show good agreement about how the effective permittivity of substrates can be influenced by adding inclusions. Future work will include continuing to work with nanomaterial experts to develop practical samples and measure the antenna radiation patterns. It is hypothesized that by strategically locating the metallic sections and varying the permittivity, that the surface waves can be reduced and hence the antenna efficiency can be improved. 


\section{ACKNOWLEDGEMENTS}

This work was supported by EPSRC under Grant No. EP/I01490X/1.

\section{REFERENCES}

[1] L. J. Chu, "Physical limitations on omni-directional antennas," Journal of Applied Physcis, vol. 19, pp. 13691393, 1948.

[2] H. A. Wheeler, "Fundamental Limitations of Small Antennas," Proceedings of the IRE, vol. 35, pp. 1479-1484, 1947.

[3] J. Volakis, Antenna Engineering Handbook, 4th ed: McGraw Hill, 2007.

[4] J. R. James and P. S. Hall, Handbook of Microstrip Antennas: IET, 1989.

[5] J. R. James, P. S. Hall, and C. Wood, Microstrip Antenna Theory and Design: Peter Peregrinus Ltd, 1986.

[6] P. S. Hall, "The contribution of J R James to printed antennas," presented at The Second European Conference on Antennas and Propagation (EuCAP 2007) Edinburgh, UK, 2007.

[7] W. G. Whittow, C. C. Njoku, and Y. C. Vardaxoglou, "Patch Antennas with Heterogeneous Substrates and Reduced Material Consumption Enabled by Additive Manufacturing Techniques," presented at 2012 IEEE International Symposium on Antennas and Propagation and USNC-URSI National Radio Science Meeting, Chicago, USA, 2012.

[8] M. Baumers, C. J. Tuck, and R. J. M. Hague, "Realised levels of geometric complexity in additive manufacturing," International Journal of Product Development, vol. 13, pp. 222-244, 2011.

[9] G. Kiziltas, D. Psychoudakis, and J. L. Volakis, "Topology design optimization of dielectric substrates for bandwidth improvement of a patch antenna," Antennas and Propagation, IEEE Transactions on, vol. 51, pp. 2732-2743, 2003.

[10] D. Psychoudakis, Y. H. Koh, J. L. Volakis, and J. H. Halloran, "Design method for aperture-coupled microstrip patch antennas on textured dielectric substrates," Antennas and Propagation, IEEE Transactions on, vol. 52, pp. 2763-2766, 2004.

[11] D. Psychoudakis, J. L. Volakis, W. Zach, and J. W. Halloran, "Cavity-Backed Miniature Wideband UHF Circular Polarized Antenna With Textured Dielectrics," Antennas and Propagation, IEEE Transactions on, vol. 54, pp. 3586-3592, 2006.

[12] C. Chen and J. L. Volakis, "Bandwidth broadening of patch antennas using nonuniform substrates," Microwave and Optical Technology Letters, vol. 47, pp. 421-423, 2005.

[13] M. I. Kitra, C. J. Panagamuwa, P. McEvoy, J. C. Vardaxoglou, and J. R. James, "Low SAR ferrite handset antenna design," IEEE Trans Antennas and Propagation, vol. 55, pp. 1155-1164, 2007.

[14] L. Lewin, "The electrical constants of a material loaded with spherical particles," Proceedings of the IEE, vol. 94, pp. 65-68, 1947.

[15] C. C. Njoku, W. G. Whittow, and J. C. Vardaxoglou, "Comparative study of nanomaterials' effective properties using canonical formulations," presented at 2010 Loughborough Antennas \& Propagation Conference (LAPC), Loughborough, UK, 2010.

[16] W. T. Doyle, "Optical properties of a suspension of metal spheres," Phy. Rev. B, vol. 39, pp. 9852-9858, 1989.

[17] C. C. Njoku, W. G. Whittow, and J. C. Vardaxoglou, "Simulation Methodology for Synthesis of Antenna Substrates with Micro-scale Inclusions," IEEE Trans Antennas and Propagation. In press. Accepted for publication in Nov 2011 and will be printed in 2012, 2012.

[18] N. G. Alexopoulos, C. A. Kyriazidou, and H. F. Contopanagos, "Effective parameters for metamorphic materials and metamaterials through a resonant inverse scattering approach," IEEE Trans. Microw. Theory Tech, vol. 55, pp. 254-267, 2007.

[19] C. C. Njoku, W. G. Whittow, and J. C. Vardaxoglou, "Microwave antennas and heterogeneous substrates using nanomaterial fabrication techniques (Invited Paper)," presented at IEEE Tropical Conference on Antennas and Propagation in Wireless Communications (APWC 11), Torino, Italy, 2011.

[20] C. C. Njoku, W. G. Whittow, and Y. C. Vardaxoglou, "Effective permittivity of heterogeneous substrates with cubes in a 3D lattice," IEEE Antennas and Wireless Propagation Letters (Special Issue on Metamaterials), In press, vol. Feb 2012, 2012.

[21] C. C. Njoku, W. G. Whittow, and J. C. Vardaxoglou, "Designing Microwave Patch Antennas Using Heterogeneous Substrates," to be presented at EUCAP 2012: The 6th European Conference on Antennas and Propagation, Prague, Czech Republic, 2012. 